\title{
Localized prostate cancer
}

\author{
Anil Kapoor, MD, FRCSC;' Sebastien J. Hotte, MD, FRCPC ${ }^{2}$
}

Professor of Surgery (Urology), McMaster University, Associate Editor (Oncology), Canadian Urological Association Journal (CUAJ), Hamilton, ON, Canada; ${ }^{2}$ Associate Professor, Department of Oncology, Division of Medical Oncology, McMaster University; Medical Oncologist, Juravinski Cancer Centre, Hamilton, ON, Canada

Cite as: Can Urol Assoc J 2016;10(7-8-Supp|3):S138-9. htrp://dx.doi.org/10.5489/cuai.4054

Multiparametric magnetic resonance imaging may identify one-quarter of men who might safely avoid unnecessary biopsy

In the current diagnostic pathway for prostate cancer, men with an elevated prostate-specific antigen (PSA) or other risk factors typically undergo transrectal ultrasound (TRUS)guided biopsy. However, there are several potential drawbacks - clinically indolent cancers may be identified by chance, clinically significant lesions may be missed, and important cancers may be incorrectly classified as unimportant. In many cases, men may unnecessarily undergo radical treatment to the prostate, resulting in side effects such as erectile dysfunction and incontinence. Multiparametric magnetic resonance imaging (MP-MRI) holds promise as a triage test for men with elevated PSA levels to determine the need for TRUS biopsy. The 11-centre UK Prostate MRI Imaging Study (PROMIS) compared MP-MRI and TRUS biopsy with an accurate reference test (template prostate mapping biopsy with sampling of the prostate every $5 \mathrm{~mm}$ ) in 576 men with an elevated PSA (up to $15 \mathrm{ng} / \mathrm{mL}$ ) and no prior biopsy. ${ }^{1}$ Results presented at ASCO 2016 revealed MP-MRI to be significantly more sensitive than TRUS biopsy (93\% vs. $48 \% ; p<0.0001)$, with a higher negative predictive value ( $89 \%$ vs. $74 \% ; p<0.0001)$, but lower positive predictive value $(51 \%$ vs. $90 \% ; p<0.0001)$ and specificity $(96 \%$ vs. $41 \% ; p<0.0001)$. Clinically significant disease — defined as a Gleason score of 4 or higher or a high burden of low-grade disease - was missed in 119 cases by TRUS biopsy, including 13 Gleason $4+3$ or higher cancers. MP-MRI missed only 17 cases of clinically significant disease and no Gleason $4+$ 3 or higher cancers. While TRUS-biopsy performs poorly in both detecting and ruling out clinically significant prostate cancer, MP-MRI shows promise as a triage test, with the ability to identify up to one-quarter of men who might safely avoid unnecessary biopsy, without impairing the detection of clinically significant cancer. This research adds to the growing body of evidence highlighting the role of prostate MRI in clarifying prostate cancer diagnosis.

Shorter radiation fractionation regimen is not inferior to conventional radiotherapy for intermediate-risk, localized prostate cancer

Localized prostate cancer is often treated with high-dose radiation therapy over the course of 7.5-8.5 weeks. However, the dose-response of prostate tumours and normal tissues to fractionated radiation therapy, described by the $\alpha-\beta$ ratio, is relatively low, lending to the hypothesis that hypo-fraction radiation therapy may be more effective in prostate cancer. At ASCO 2016, Dr. Charles Catton from the Princess Margaret Cancer Centre in Toronto presented results from the randomized, controlled, non-inferiority PROFIT study, which explored whether an eight-week course of escalateddose conformal radiation therapy can be safely compressed into a four-week course, with similar efficacy, in men with intermediate-risk prostate cancer. ${ }^{2}$ Over a period of four years, 1206 men from 27 centres in Canada, Australia, and France were treated with either hypofractionated $(n=608)$ or conventional radiation therapy $(n=598)$. At the time of reporting, 166 patients receiving hypofractionated radiation therapy had experienced a biochemical clinical failure (BCF) event compared with 175 in the conventional radiation therapy group, corresponding to a BCF event rate of $21 \%$ at five years in both arms (hazard ratio [HR] 0.96; 90\% confidence interval $[\mathrm{CI}]$ 0.80-1.15). There were no differences in overall survival (OS) or acute toxicity of Grade 3 or higher between the two groups. However, for gastrointestinal toxicity of Grade 2 or higher, those who underwent hypofractionated radiation therapy had significantly more acute events $(p=0.003)$ and significantly fewer late events $(p=0.006)$ than those who received conventional radiation therapy. Quality of life (QOL) measures over four years were similar between the two groups. Given the potential for cost-savings and greater convenience to the patient, these results may very well define a new standard of care for men with intermediate disease treated with external beam radiation therapy (EBRT). 
The addition of short-term androgen depletion to high-dose radiotherapy improves PSA or clinical relapse-free survival in men with localized intermediate-risk prostate cancer

Results from the final analysis of the GETUG 14 trial were presented in a poster session at ASCO 2016. This multicentre, randomized trial evaluated the addition of fourmonth androgen-deprivation therapy (ADT) to high-dose radiotherapy in 377 men with intermediate-risk localized prostate cancer. ${ }^{3}$ Patients were randomly assigned to highdose conformal radiotherapy (80 Gy), either alone or in combination with four months of ADT consisting of flutamide plus triptorelin. After a median followup of 84 months, the group that received both radiotherapy and ADT had a significantly higher rate of clinical/biochemical relapsefree survival compared with those who received radiation therapy alone ( $84 \%$ vs. $76 \% ; p=0.02)$, as well as a lower cumulative incidence of biochemical failure $(10 \%$ vs. $21 \%$; $\mathrm{p}<0.01)$. There was no difference in OS between the two groups (93\% vs. 94\%; $p=0.54$ ). Moderate liver toxicity was more frequent in the ADT group.

Statin use is associated with improved biochemical recurrencefree survival in men with prostate cancer who underwent radical prostatectomy for curative intent

In addition to their positive effects on hypercholesterolemia, cardiovascular events, and mortality, statins have recently received attention for their potential anticancer properties. Trapp and colleagues presented their poster at ASCO 2016, summarizing preliminary results of their study on the effects of statins on rates of biochemical recurrence-free (BCRF) survival in 3311 men with prostate cancer who underwent radical prostatectomy for curative intent at the Robert $\mathrm{H}$. Lurie Comprehensive Cancer Center at Northwestern University, Chicago between 2002 and 2005. ${ }^{4}$ Patients were stratified according to their statin use within two years before or any time after undergoing radical prostatectomy. After a median followup of 49.0 months (range 1-150 months), the five-year BCRF survival rate was significantly longer among the 1023 statin users than among the 2288 non-statin users (58.2\% vs. $52.7 \%$; log-rank $p<0.01$ ). All of the patients in both groups were still alive at five years. This abstract adds to the growing body of research illustrating the benefits of statin use in decreasing malignancy rates.
Radical prostatectomy is associated with higher early mortality, but better long-term survival than external beam radiation therapy in men with stage II prostate cancer

In a recent study comparing five-year progression-free probability in 13803 men who underwent radical prostatectomy, EBRT, or brachytherapy, prostate cancer-specific mortality was higher with EBRT than with radical prostatectomy. ${ }^{5}$ However, a recent systematic review could not provide strong evidence to support one therapy over another for the treatment of localized prostate cancer. ${ }^{6}$ Using the United States National Cancer Database, Marsh and colleagues compared survival in 455106 men with stage II prostate cancer treated with either EBRT $(n=169$ 185) or radical prostatectomy $(\mathrm{n}=285921) .^{7}$ Patients who underwent radical prostatectomy were on average younger than those who underwent EBRT (60.56 years vs. 69.42 years; $\mathrm{p}<0.05)$ and had a higher average PSA level $(11.82 \mathrm{ng} / \mathrm{mL}$ vs. $7.67 \mathrm{ng} / \mathrm{mL} ; \mathrm{p}<0.05)$. White men were more likely to receive radical prostatectomy than non-White men $(64.5 \%$ vs. $35.5 \% ; p<0.05)$. Unadjusted mean survival was significantly longer for men who underwent radical prostatectomy than for those who underwent EBRT (124.5 months vs. 106.5 months; $\mathrm{p}<0.05)$. Longer survival was also observed for men who were younger, White, had a lower Charlson/ Deyo score, and lower PSA levels. Although early mortality was higher among the men treated with radical prostatectomy, this group had significantly better long-term survival. In multivariate analysis, OS was superior in the EBRT group at three years (HR 2.12; $\mathrm{p}<0.05)$; however, at 10 years, OS favoured radical prostatectomy (HR 0.11; $\mathrm{p}<0.05)$.

\section{References}

1. Ahmed HU, El-Shater Bosaily A, Brown LC, et al. The PROMIS study: A paired-cohort, blinded confirmatory study evaluating the accuracy of multiparametric MRI and TRUS biopsy in men with an elevated PSA. J Clin Oncol 2016;34(suppl). Abstr 5000.

2. Catton $\mathrm{CN}$, Lukka H, Julian JA, et al. A randomized trial of a shorter radiation fractionation schedule for the treatment of localized prostate cancer. J Clin Oncol 2016;34(suppl). Abstr 5003.

3. Dubray BM, Salleron J, Gilles $S$, et al. Does short-term androgen depletion add to high-dose radiotherapy ( $80 \mathrm{~Gy}$ ) in localized intermediate risk prostate cancer? Final analysis of GETUG 14 randomized trial (EU-20503/NCT00104741). J Clin Oncol 2016;34(suppl). Abstr 5021.

4. Trapp L, Helenowski IB, Gurley M, et al. Use of statins and the risk of prostate cancer biochemical recurrence in men treated with radical prostatectomy. J Clin Oncol 2016;34(suppl). Abstr 5048.

5. Lee BH, Kibel AS, Ciezki JP, et al. Are biochemical recurrence outcomes similar after radical prostatectomy and radiation therapy? Analysis of prostate cancer-specific mortality by nomogram-predicted risks of biochemical recurrence. Eur Urol 2015;67:204-9. http://dx.doi.org/10.1016/i.eururo.2014.09.017

6. Wolff RF, Ryder $S$, Bossi A, et al. A systematic review of randomized, controlled trials of radiotherapy for localized prostate cancer. Eur J Cancer 2015;51:2345-67. http://dx.doi.org/10.1016/i. ejcc.2015.07.019

7. Marsh S, Walters RW, Silberstein PT. Overall survival in stage II prostate cancer after radiation or surgical therapy. J Clin Oncol 2016;34(suppl). Abstr 5068. 